\title{
The apparent secrets of the structure and functions of mitochondrial processes in the cells at all endogenous levels in the light of the clinical effectiveness of the molecular method of biocybernetic clavitherapy
}

\section{Conceptual paper}

Claims by some scientists of molecular and cellular biology that without the full understanding of processing occurring in the cells in the bodies of people suffering from entirely drug-resistant incurable diseases, that there are no effective methods of enhancement are untrue. Research in leading worldly laboratories equipped with the state-ofthe-art diagnostic/testing apparatus show the energy productivity of the human body with chronic health exhaustion as well as an electron microscope, which magnifies 5million times. Carrying out a scientific programme oriented at the structure and functions of mitochondria in the energy processes occurring at subcellular level in the cells and tissues of the human body called "cellular power stations" are not completely consistent with many years' clinical investigations and achievements of the molecular method of biocybernetic clavitherapy.

As we know from the publications of prestigious scientific periodicals the cells of more complicated eukaryotic organisms have mitochondria, where cellular breathing occurs carried by the forced quality of blood supply with clavitherapy procedures in the dermatome and connective tissue and other endogenous levels along with clavitherapy exercises. In the clinical conditions of applying clavitherapy It was established that patients with severe chronic ailments with very limited physiological productivity obtain, after a short cure, very good results of medical tests with sensational mental and physical fitness.

Disorders caused by mitochondrial diseases are relatively rare and frequently have an acute course leading to death not only at a child's age. As some scientific sources state their genetic basis is lack of the function of mitochondrial proteins, most frequently involved in cellular breathing and the production of energy.

It is widely known that mitochondria supply organisms not only with energy but also participate in the production of 3 million molecular endogenous factors and around 10million submolecular transmitters and neurotransmitters. It happens so in the organism when defects in mitochondria disturbed by the excessive use of chemicals of our civilization, disturbed from physical laziness and enzymatic and metabolic functions lead to very grave consequences linked with an energy deficit and excessive effects of supmetabolic wastes. Mitochondrial diseases regard the cells and tissues neglected at all endogenous levels dependent on good blood supply.

Even if we have not explored and do not fully comprehend the processes responsible for the proper functioning of mitochondria, there is a tested possibility of restoring the proper processes at subcellular level of all endogenous systems. It includes mitochondrial processes
Volume 3 Issue 6 - 2016

Ferdynand Barbasiewicz

Ferdynand Barbasiewicz, The creator of the method, Poland

Correspondence: Ferdynand Barbasiewicz, The creator of the method, Poland, Tel +48227298053 Email centrum@klawiterapia.com

Received: July 12, 2016 | Published: July 13, 2016

besides some extremely emaciated organisms with substantial genetic deficiencies and in advanced negligence of old age. The clavitherapy method allows reconstructing in 7-10 days immunological resistance lost largely in aenemia, leukopenia and thrombocytopenia. After a short (7-10 days) series of 2- or 3-hour clavitherapy procedures patients with chronic and drug-resistant diseases leave the Centre of Clavitherapy fresh as a daisy. They are fully fit and their energy and fitness manifest themselves in many physical actions and also in sexual intercourses without becoming fatigued with a strong volitional factor and measurable satisfaction even at elderly age. Daily clavitherapy exercises performed in the evening and morning and sex at a minimum of twice a week preserving the rules of partnership, hygiene with a dose of fantasy is the main drive of maintaining constant mitochondrial activity in the cells at all endogenous levels, even at advanced age.

\section{To whom it may concern}

Like antibiotics, the latest steroids and other medications and physiotherapy do not help. I propose employing the cellular treatment of clavitherapy in the reconstruction of immunological resistance. As I mentioned according to American research 3million various sorts of molecular endogenous factors circulate in the blood of a healthy person, including around 100 thousand selective antibodies (established by me, I have clinical evidence). Moreover, there are around 10million submolecular neurochemical transmitters and neurotransmitters in the blood. All molecular factors, antibodies and submolecular neurochemical transmitters are controlled biocybernetically noninvasively through dermal receptors, collagen innervated fibres, numerous biologically active points, also cryogenic, acupuncture ones and the nervous system. The entire method of clavitherapy, which I have been investigating and experimenting with for over 50years with verification in clinical conditions is formulated methodically by me in 1100 algorithms with the network of actions quantified with medical diagnosis for various functional dysfunctions and diseases described and expressed in the books: Clavitherapy and the Atlas 
of Clavitherapy. To characterize the method of clavitherapy most generally it is essential to recognize as art stimulation with clavics, like lat. clavus $=$ nail in controlling dermovisceral repair processes of molecular, physiological and psychosomatic disturbances and out of negligence and ischemia due to physical exercise, traumas and infection of homeostasis of the old age of the human body. The other clinical achievement and even my discovery was the precise etiological specification of functional and structural disorders of the nervous system forming the main causes of many various drug-resistant ailments and in rehabilitation. In the 80 s in Klinika Neurologiczna Szpitala Klinicznego MSW (Neurological Clinic of the Clinical Hospital of the Ministry of Internal Affairs) in Warsaw I developed an effective and simple method of liquidating in several days demyelinising disturbances in the brain and in various sorts of peripheral nervous fibres and neuron palsies with glioblastomas elicited by the earliest changes of the malignant neoplasm, which are entirely drug-resistant. The range of effectiveness of clavitherapy is expressed in short in the publication entitled Molecular medicine of the distant future available just today. It also concerns resuscitation without the defibrillator and massaging the chest. Presently there are several masters of clavitherapy and there are two people admitted into a doctoral programme at medical universities. The fourth edition of Clavitherapy and the Atlas of Clavitherapy in 3 volumes are already prepared in Polish and will be printed towards the end of September this year. Awaiting in the near future the successful incorporation of the method of clavitherapy into education, research and specialization programmes at medical universities, medicine-related ones and higher education institutions about health. I kindly ask you to become acquainted with the stance of Polskie Towarzystwo Lekarskie (Polish Doctors' Society). Please read the attached publications. Please watch short videos at: ATVN on www.klawiterapia.com and the PTL board's opinion www.youtabe.com/wath? $=y m Z O f e Y L D t k$ www. instytutklawiterapii.com/yt

\section{Acknowledgements}

None.

\section{Conflict of interest}

The author declares no conflict of interest. 